ISSN 0103-5150

Fisioter. Mov., Curitiba, v. 25, n. 4, p. 849-854, out./dez. 2012

Licenciado sob uma Licença Creative Commons

\title{
Efeitos agudos de uma sessão de reabilitação cardíaca sobre a pressão arterial
}

\author{
Acute effects of one session of cardiac \\ rehabilitation on arterial blood pressure
}

\section{Murillo Frazão de Lima e Costa ${ }^{[a]}$, Danielly Inocêncio de Araújo ${ }^{[b]}$}

[a] Fisioterapeuta, mestrando em Fisioterapia na Universidade Federal do Rio Grande do Norte (UFRN), especialista em Fisioterapia Pneumofuncional pela Universidade de Brasília (UnB), especialista em Reabilitação Cardíaca pela Universidade Gama Filho, Natal, RN - Brasil, e-mail: murillo.frazao@gmail.com

[b] Fisioterapeuta, especialista em Fisioterapia Cardiorrespiratória pelo Centro Universitário de João Pessoa (UNIPÊ), João Pessoa, PB - Brasil, e-mail: nelly_fisio@yahoo.com.br

\section{Resumo}

Introdução: Hipertensão arterial é uma condição mórbida presente em grande parte dos adultos. Estudos evidenciam que exercícios físicos de moderada intensidade e curta duração têm efeitos satisfatórios na redução da pressão arterial. Objetivo: Mensurar a duração do efeito hipotensor de uma única sessão de reabilitação cardíaca a partir da prática de exercício aeróbico de curta duração e moderada intensidade. Materiais e métodos: 0 estudo foi prospectivo e quase-experimental. Participaram do estudo nove voluntários hipertensos e sedentários (cinco do sexo feminino e quatro do masculino), monitorizados pelo exame de medida ambulatorial da pressão arterial por 12 horas e 30 minutos. Foi realizada uma única sessão de treinamento aeróbico por dez minutos em bicicleta ergométrica numa intensidade moderada. Resultados: Houve redução tanto na PAS (pressão arterial sistólica) como na PAD (pressão arterial diastólica) (ANOVA, p < 0,001) durante as 12 horas de estudo. Conclusão: Uma sessão de exercício físico de curta duração com intensidade moderada foi suficiente para reduzir os níveis pressóricos dos voluntários analisados por um período de 12 horas.

Palavras-chave: Treinamento aeróbico. Hipotensão. Pressão arterial. 


\begin{abstract}
Introduction: Hypertension is a morbid condition present in most adults. Studies show that exercise of moderate intensity and short duration has satisfactory effects on lowering blood pressure. Objective: To measure the duration of the hypotensive effect of a single session of cardiac rehabilitation, through aerobic exercise of short duration and moderate intensity. Materials and methods: The study was prospective and quasi-experimental. Study participants were nine hypertensive and sedentary volunteers (five female and four male), monitored by examining measured ambulatory blood pressure for 12 hours and 30 minutes. We performed a single session of aerobic training for ten minutes on a stationary bicycle at a moderate intensity. Results: There was a reduction in both SBP (systolic blood pressure) and DBP (diastolic blood pressure) (ANOVA, $p<0.001$ ) during the 12 hours of study. Conclusion: One session of short duration exercise at moderate intensity was sufficient to reduce blood pressure levels of the volunteers studied for a period of 12 hours.
\end{abstract}

Keywords: Aerobic training. Hypotension. Blood pressure.

\section{Introdução}

Hipertensão arterial é uma condição mórbida e um dos principais fatores de risco para o desenvolvimento de doenças cardiovasculares em adultos. Uma análise durativa realizada por dez anos revelou a incidência de doenças cardiovasculares em adultos hipertensos entre 35 e 64 anos em 4\% dos indivíduos do gênero feminino e em $8 \%$ do gênero masculino; na faixa etária de 65 a 90 anos, esses índices sobem para 19\% dos indivíduos do gênero feminino e 25\% do masculino (1).

O número de hipertensos tem crescido muito nos últimos anos, principalmente na população idosa, o que aumenta a incidência de doenças cardiovasculares. Fatores de risco como obesidade e ingestão de bebida alcoólica, comuns na população atual, aumentam o risco de incidência de desenvolvimento de hipertensão arterial em 1,56 e 1,22 vezes, respectivamente $(2,3)$.

Durante e após a realização de atividade física, a necessidade de resfriamento corporal e aumento do aporte sanguíneo para os músculos esqueléticos promovem uma inibição do sistema nervoso simpático; como consequência, ocorre vasodilatação periférica e diminuição da resistência vascular e da pressão arterial (4-7).

Estudos que avaliam exercício físico como tratamento não medicamentoso para hipertensão arterial indicaram que ocorre boa resposta terapêutica. Muitos desses efeitos ocorrem entre a primeira e a quinta sessão, o que indica que a curva dose-resposta pode ser abrupta (8). Estudos como o de MacDonald et al. (9) demonstram que exercícios aeróbicos, de moderada intensidade realizados em cicloergômetros, são suficientes para causar um efeito hipotensor, e que 10 ou 30 minutos de exercício promovem a mesma resposta hipotensora. A monitorização contínua da medida ambulatorial de pressão arterial indica que esses efeitos hipotensores agudos podem perdurar por horas (10).

Assim, o objetivo do presente estudo é mensurar a duração do efeito hipotensor de uma única sessão de reabilitação cardíaca a partir da prática de exercício aeróbico de curta duração e moderada intensidade.

\section{Materiais e métodos}

O estudo foi prospectivo e quase-experimental. 0 trabalho foi aprovado pelo Comitê de Ética em Pesquisa da Secretaria Estadual de Saúde da Paraíba (CEP/SES-PB). Participaram do estudo nove voluntários hipertensos e sedentários. Os voluntários foram monitorizados pelo exame de medida ambulatorial da pressão arterial (MAPA Pro Medic) por 12 horas e 30 minutos (período de vigília); o aparelho foi programado para gravar dados pressóricos a cada 15 minutos (totalizando 50 aferições de forma sequencial). Após a instalação do MAPA, os voluntários se mantiveram em repouso, sentados, durante três aferições de pressão arterial (totalizando 30 minutos).

Logo em seguida, foi realizada uma única sessão de treinamento aeróbico por dez minutos em bicicleta ergométrica (Reebok Power Bike), numa intensidade moderada (60-70\% da frequência cardíaca máxima prevista para cada indivíduo e Escala de Esforço Subjetivo de Borg adaptada numa faixa de 6 a 8). 
Após a realização da atividade física, os pacientes se mantiveram na posição inicial de repouso (sentados) durante uma aferição de pressão arterial (quarta aferição) e, em seguida, foram encaminhados aos seus domicílios e orientados a permanecerem em repouso (sentados ou deitados) durante as 12 horas de monitorização. Caso não pudessem permanecer em repouso, os pacientes deveriam registrar a atividade realizada em diário oferecido. A análise dos dados obtidos no estudo foi realizada com o uso do software "Graph Pad Prism 4"; para determinação de diferenças estatísticas com valor de significância p < 0,05 foi empregados o teste de normalidade ShapiroWilk e o método ANOVA.

\section{Resultados}

As características dos voluntários - idade, gênero, índice de massa corporal e medicameno anti-hipertensivo utilizado - estão descritas no Quadro 1, a seguir:

0 grupo analisado foi aprovado no teste de normalidade Shapiro-Wilk (alpha $=0.05$ ). A PAS média foi de 157,5 $\pm 16,98 \mathrm{mmHg}$ e PAD média foi de 93,11 $\pm 14,10 \mathrm{mmHg}$ no início do estudo. Houve redução tanto na PAS como na PAD durante as 12 horas de estudo (Figuras 1 e 2).

Quadro 1 - Características dos voluntários

\begin{tabular}{ccccl}
\hline Voluntário & Idade & Gênero & IMC & $\begin{array}{l}\text { Droga anti-hiper- } \\
\text { tensiva em uso }\end{array}$ \\
\hline 1 & 56 & $\mathrm{~F}$ & 32,81 & Captopril \\
2 & 65 & $\mathrm{~F}$ & 22,43 & Losartana Potássica \\
3 & 65 & $\mathrm{~F}$ & 26,3 & Captopril \\
4 & 69 & $\mathrm{~F}$ & 32,81 & Losartana Potássica \\
5 & 68 & $\mathrm{M}$ & 25,86 & Captopril \\
6 & 57 & $\mathrm{M}$ & 25,58 & Nenhuma \\
7 & 30 & $\mathrm{M}$ & 30,12 & Nenhuma \\
8 & 79 & $\mathrm{~F}$ & 22,22 & Losartana Potássica \\
9 & 78 & $\mathrm{M}$ & 24,01 & Nenhuma \\
\hline
\end{tabular}

Legenda: IMC = índice de massa corporal.

Fonte: Dados da pesquisa.

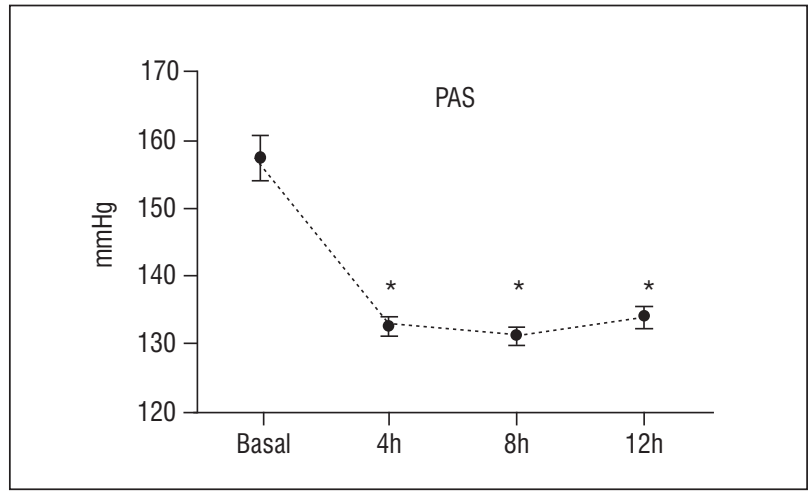

Figura 1 - Comportamento da PAS durante 12 horas após 0 exercício ( $* p<0,001$ comparada à basal)

Fonte: Dados da pesquisa.

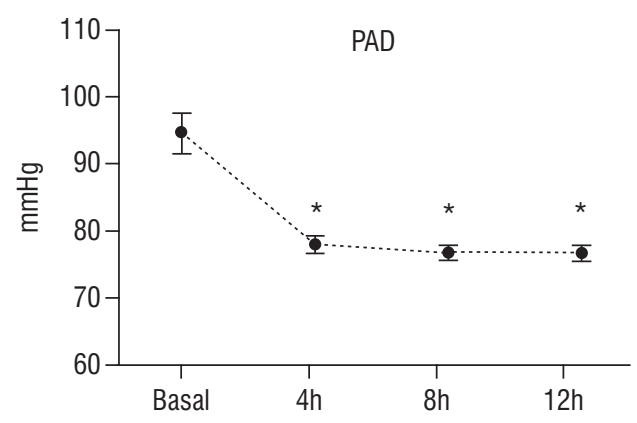

Figura 2 - Comportamento da PAD durante 12 horas após 0 exercício (* $p<0,001$ comparada à basal)

Fone: Dados da pesquisa.

A PAS média durante as 4 primeiras horas pós-exercício foi de 132,7 $\pm 17,51 \mathrm{mmHg}$ ( $\mathrm{p}<0,001 \mathrm{com}$ paradaa à basal) e a PAD foi de 77,09 $\pm 13,96 \mathrm{mmHg}$ $(\mathrm{p}<0,001$ comparada à basal). A PAS entre 4 e 8 horas pós-exercício foi de 131,1 $\pm 16,95 \mathrm{mmHg}(\mathrm{p}<0,001$ comparada à basal) e a PAD foi de 75,92 $\pm 12,92 \mathrm{mmHg}$ ( $p<0,001$, comparada à basal). A PAS entre 8 e 12 horas pós-exercício foi de 133,9 $\pm 17,82 \mathrm{mmHg}(\mathrm{p}<0,001$ comparada à basal) e a PAD foi de 75,84 $\pm 12,14 \mathrm{mmHg}$ ( $p<0,001$ comparada à basal). Não foram observadas diferenças significativas nas aferições da PAS e da PAD nos períodos entre 4, 8 e 12h após o exercício.

\section{Discussão}

A atividade física contribui para a redução da pressão arterial porque aumenta a atividade do sistema 
nervoso parassimpático e inibe a atividade simpática (6), melhorando a sensibilidade de barorreceptores (1113); além disso, a atividade física influencia a função endotelial $(14,15)$, aumentando a complacência arterial.

Para ser clinicamente relevante, a hipotensão pós-exercício necessita ter uma magnitude significante e ser sustentada por um longo período sob condições ambulatoriais (16). Como demonstrado no presente estudo, foi significativa a redução pressórica observada após uma única sessão de dez minutos de atividade aeróbica; vale salientar como efeito benéfico que essa redução foi sustentada por 12 horas. Outros trabalhos relatam redução da pressão arterial por até 24 horas (17).

Revisões sobre o tema $(18,19)$ examinaram o tempo da hipotensão pós-exercício usando monitorização ambulatorial, porém os resultados são contraditórios, porque possivelmente o período pós-exercício não foi bem controlado em todos os estudos.

Hagberg (20) refere que há evidências de que pacientes sedentários hipertensos apresentam significativa redução na pressão arterial, especialmente na PAS. Essa redução fica evidenciada por um grande período de tempo após uma única sessão de exercício submáximo, corroborando com os dados encontrados no presente estudo.

Embora existam diferenças entre os gêneros no que diz respeito à pressão arterial e atividade simpática, a hipotensão encontrada foi similar entre os grupos. Esse resultado ratifica, portanto, estudos realizados entre ambos os gêneros $(21,22)$ e grupos específicos (23).

Com relação à intensidade do exercício, o presente estudo demonstrou que exercícios moderados são suficientes para diminuir os níveis pressóricos. Esse resultado ratifica o estudo de Viecili et al. (24), que demonstrou eficácia na diminuição da pressão arterial já na primeira sessão com 20 minutos de duração, também com intensidade moderada. Isso indica que é possível obter resultados satisfatórios com níveis mais seguros de exercício, contrariamente aos achados de Quinn et al. (25), em que foi evidenciada redução absoluta maior e mais duradoura da pressão arterial com exercícios de intensidade elevada com 30 minutos de duração.

Em outro trabalho (26), que utilizou o exercício com 50 minutos de duração e intensidade de $50 \%$ do VO2 (consumo máximo de oxigênio) para demonstrar os efeitos hipotensores do exercício, observaram-se reduções significativas na PAS e na PAD durante o período diurno, de 11 e 4 horas, respectivamente.
Semelhante resultado ocorreu na pesquisa de Taylor (27), em que ocorreu a hipotensão após exercício moderado de 30 minutos, sendo a PAS reduzida por até 16 horas e a PAD por quatro horas.

Quanto à duração do exercício, Mac Donald (28) documentou que dez minutos de exercício moderado foram suficientes para eliciar uma diminuição da pressão arterial sistólica e diastólica pós-exercício em indivíduos hipertensos. Entretanto, a mensuração da pressão, diferentemente do presente estudo, foi mensurada somente até uma hora após a realização do exercício. Outro estudo (29) demonstrou que exercícios de maior duração têm efeitos hipotensores mais acentuados, quando comparados a exercícios de menor duração.

\section{Conclusão}

O presente estudo observou que uma sessão de exercício físico de curta duração com intensidade moderada foi suficiente para reduzir os níveis pressóricos dos voluntários analisados por um período de 12 horas. Esse tipo de terapia pode ser utilizada como método não farmacológico para o tratamento da hipertensão arterial.

\section{Referências}

1. Vasan RS, Larson MG, Leip EP, Evans JC, O'Donnell CJ, Kannel WB, et al. Impact of high-normal blood pressure on the risk of cardiovascular disease. N Engl J Med. 2001;345(18):1291-7. doi:10.1056/NEJMoa003417.

2. Motlagh B, O'Donnell M, Yusuf S. Prevalence of cardiovascular risk factors in the middle east: a systematic: review. Eur J Cardiovasc Prev Rehabi. 2009;16(3):26880. doi:10.1097/HJR.0b013e328322ca1b.

3. Wang W, Lee ET, Fabsitz RR, Devereux R, Best L, Welty TK, et al. A longitudinal study of hypertension risk factors and their relation to cardiovascular disease: the strong heart study. Hypertension. 2006;47(3):403-9. doi:10.1161/01.HYP.0000200710.29498.80.

4. Chen CY, Bechtold AG, Tabor J, Bonham AC. Exercise reduces GABA synaptic input onto nucleus tractus solitarii baroreceptor second-order neurons via nk1 receptor internalization in spontaneously hypertensive rats. J Neurosci. 2009;29(9):2754-61. doi:10.1523/ JNEUROSCI.4413-08.2009. 
5. Franklin PJ, Green DJ, Cable NT. The influence of thermoregulatory mechanisms on post-exercise hypotension in humans. J Physiol. 1993;470:231-41. PMid:8308728.

6. Casonatto J, Tinucci T, Dourado AC, Polito M. Cardiovascular and autonomic responses after exercise sessions with different intensities and durations. Clinics. 2011;66(3):453-8. doi:10.1590/ S1807-59322011000300016.

7. Cucato GG, Ritti-Dias RM, Wolosker N, Santarem JM, Jacob W Filho, Forjaz CL. Post-resistance exercise hypotension in patients with intermittent claudication. Clinics. 2011;66(2):221-6. doi:10.1590/ S1807-59322011000200007.

8. Viecili PRN, Bundchen DC, Richter CM, Dipp T, Lamberti DB, Pereira AMR, et al. Curva dose-resposta do exercício em hipertensos: análise do número de sessões para efeito hipotensor. Arq Bras Cardiol. 2009;92(5):393-9. doi:10.1590/S0066-782X2009000500010.

9. MacDonald JR, MacDougall JD, Hogben CD. The effects of exercise duration on post-exercise hypotension. J Hum Hypertens. 2000;14:125-9. doi:10.1038/ sj.jhh.1000953.

10. Taylor-Tobert NS, Dengel DR, Brown MD, McCole SD, Pratley RE, Ferrel RE, et al. Ambulatory blood pressure after acute exercise in older men with essential hypertension. J Hum Hypertens. 2000;13(1 Pt 1):44-51.

11. La Rovere MT, Bersano C, Gnemmi M, Specchia G, Schwartz PJ. Exercise-induced increase in baroreflex sensitivity predicts improved prognosis after myocardial infarction. Circulation. 2002;106(8):945-9. doi:10.1161/01.CIR.0000027565.12764.E1.

12. Madden KM, Lockhart C, Potter TF, Cuff D. Aerobic training restores arterial baroreflex sensitivity in older adults with type 2 diabetes, hypertension, and hypercholesterolemia. Clin J Sport Med. 2010;20(4):312-7. doi:10.1097/JSM.0b013e3181ea8454.

13. Willie CK, Ainslie PN, Taylor CE, Jones H, Sin PY, Tzeng YC. Neuromechanical features of the cardiac baroreflex after exercise. Hypertension. 2011;57(5):927-33. doi:10.1161/HYPERTENSIONAHA.110.164616.

14. Okada S, Hiuge A, Makino H, Nagumo A, Takaki H, Konishi $\mathrm{H}$, et al. Effect of exercise intervention on endothelial function and incidence of cardiovascular disease in patients with type 2 diabetes. J Atheroscler Thromb. 2010;17:828-33. doi:10.5551/jat.3798.
15. Vona M, Codeluppi GM, Iannino T, Ferrari E, Bogousslavsky J, von Segesser LK. Effects of different types of exercise training followed by detraining on endothelium-dependent dilation in patients with recent myocardial infarction. Circulation. 2009;119(12):16018. doi:10.1161/CIRCULATIONAHA.108.821736.

16. Cardoso CG Jr, Gomides RS, Queiroz AC, Pinto LG, da Silveira Lobo F, Tinucci T, et al. Acute and chronic effects of aerobic and resistance exercise on ambulatory blood pressure. Clinics. 2010;65(3):317-25. doi:10.1590/S1807-59322010000300013.

17. Ciolac EG, Guimarães GV, D’Ávila VM, Bortolotto LA, Doria EL, Bocchi EA. Acute aerobic exercise reduces 24-h ambulatory blood pressure levels in long-term-treated hypertensive patients. Clinics. 2008;63(6):7538. doi:10.1590/S1807-59322008000600008.

18. MacDonald JR. Potential causes, mechanisms, and implications of post exercise hypotension. J Hum Hypertens. 2002;16(4):225-36. doi:10.1038/sj.jhh.1001377.

19. Anunciacao PG, Polito MD. Hipotensão pós-exercício em indivíduos hipertensos: uma revisão. Arq Bras Cardiol. 2011;96(5):425-6. doi:10.1590/ S0066-782X2011005000025.

20. Hagberg JM, Park JJ, Brown MD. The role of exercise training in the treatment of hypertension- An update. Sports Med. 2000;30(3)193-206. doi:10.2165/ 00007256-200030030-00004.

21. Rueckert PA, Slane PR, Lillis DL, Hanson P. Hemodynamic patterns and duration of post-dynamic exercise hypotension in hypertensive humans. Med Sci Sports Exerc. 1996;28(1):24-32. doi:10.1097/ 00005768-199601000-00010.

22. Raglin JS, Turner PE, Eksten F. State anxiety and blood pressure following 30 minutes of leg ergometry or weight training. Med Sci Sports Exerc. 1993;25(9):10448. doi:10.1249/00005768-199309000-00012.

23. Paulev PE, Jordal R, Kristensen O, Ladefoged J. Therapeutic effect of exercise on hypertension. Eur J Appl Physiol Occup Physiol. 1984;53(2):180-5. doi:10.1007/BF00422584.

24. Viecili PRN, Bundchen DC, Richter CM, Dipp T, Lamberti DB, Pereira AM, et al. Curva-dose resposta do exercício em hipertensos: análise do número de sessões para efeito hipotensor. Arq Bras Cardiol. 2009;92(5):393-9. doi:10.1590/S0066-782X2009000500010. 
25. Quinn TJ. Twenty-four hour, ambulatory blood pressure responses following acute exercise: impact of exercise intensity. J Hum Hypertens. 2000;14(9):54753. doi:10.1038/sj.jhh.1001106.

26. Wallace JP. The magnitude and duration of ambulatory blood pressure reduction following acute exercise. J Hum Hypertens. 1999;13(6):361-6. doi:10.1038/ sj.jhh.1000797.

27. Taylor-Tolbert NS, Dengel DR, Brown MD, McCole SD, Pratley RE, Ferrell RE, et al. Ambulatory blood pressure after acute exercise in older men with essential hypertension. J Hum Hypertens. 2000;13(1Pt 1):44-51.

28. Mac Donald JR, Mac Dougall JD, Hogben CD. The effects of exercise duration on post-exercise hypotension. J Hum Hypertens. 2000;14(2):125-9. doi:10.1038/sj.jhh.1000953.
29. Forjaz CL, Santaella DF, Rezende LO, Barretto AC, Negrão CE. Effect of exercise duration on the magnitude and duration of post-exercise hypotension. Arq Bras Cardiol. 1998;70(2):99-104. doi:10.1590/ S0066-782X1998000200006.

Recebido: 19/08/2011 Received: 08/19/2011

Aprovado: 27/04/2012

Approved: 04/27/2012 